\title{
CORRELACIÓN DE VARIABLES ACADÉMICO-LABORALES DE EGRESADOS DE CIENCIAS CONTABLES DE LA UNMSM
}

\author{
CORRELATION OF ACADEMIC AND LABOUR VARIABLES OF \\ GRADUATES OF ACCOUNTING SCIENCE OF UNMSM
}

\author{
Ana María GutiérRez HubY* \\ Docente Principal de la Facultad de Ciencias Contables \\ Colaboradora: Iris Aguilar Alonso* \\ Universidad Nacional de San Marcos-UNMSM \\ Lima-Perú \\ [Recepción: Setiembre de 2012 / Conformidad: Octubre de 2012]
}

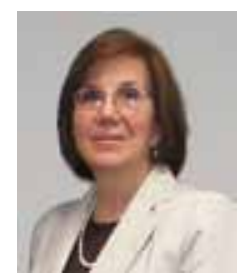

\section{RESUMEN}

El análisis que presenta este artículo, con enfoque cuantitativo, es descriptivo-explicativo por que analiza la relación entre variables de niveles de satisfacción de los egresados de la Facultad de Ciencias Contables de nuestra Universidad -a partir del sexo y experiencia laboral- con respecto a la formación profesional, desempeño y condiciones laborales actuales. Al que se aplicó la prueba estadística de independencia ChiCuadrado, la cual permitió la comprobación de las hipótesis formuladas.

Los datos obtenidos se recolectaron a través una "Encuesta del Egresado "aplicada por la Escuela Académico-Profesional de Contabilidad en el transcurso del año 2012, periodo en que se tienen registrados tres grupos de encuestados que hacen una población de 203 egresados.

A modo genérico se concluye que la presencia de mujeres en la carrera profesional contable es mayor (65\%), respecto a los varones $(35 \%)$ y que el índice de empleabilidad de los egresados (91\%), es bastante alto.

\section{ABSTRACT}

This study is part of applied research based on analysis of Comparative Financial Statements at December 31,2011-2010, published by the Lima Stock Exchange, Peru.

The cement sector is currently the most prominent on the national economy, driven by the private sector, the engine of the expansion of the sector, multifamily housing, shopping malls, supermarkets, hotels, and increased public investment, including construction and rehabilitation of roads, highways, hospitals, schools and other works in development and implementation

The analysis is performed descriptive-explanatory documentary from published financial statements and reports of local rating agencies. The objectives in developing this work are:

a) To examine whether the cement industry in Peru, is competitive and contributes to cost reduction.

b) Check if the cement industry benefited from th

Keywords: Competitiveness. Investment and Financing Structure of Economic Value Added.

* Magister en Gestión Empresarial- UNMSM, Licenciada en Ciencias Administrativas. Docente investigadora de la Facultad de Ciencias Contables- UNMSM. E-mail: anamaria01053@yahoo.es 
Para toda institución universitaria el desempeño profesional y el nivel de aceptación que tengan sus egresados en el mercado laboral debería ser un tema de vital relevancia pues la percepción positiva o negativa que se tenga de ellos respecto a su desenvolvimiento profesional implicará una respuesta clara del impacto alcanzado por parte de la institución académica en el medio laboral.

En este sentido los procesos de seguimiento al egresado constituyen una de las mejores estrategias institucionales para mantenerse con pertinencia pues son ellos los que en gran parte contribuyen a la mejora y vigencia de los contenidos curriculares, a través de la retroalimentación que mediante sus opiniones o sugerencias se produce, la cual es de gran valor porque refleja la confrontación de su formación con la realidad, adquiriendo o complementando con la práctica misma los conocimientos adquiridos en su formación académico profesional.

En el caso de la Facultad de Ciencias Contables de la Universidad Nacional Mayor de San Marcos se sigue una trayectoria en cuanto a la actividad de tomar en cuenta la opinión de sus egresados -lo ha sido tanto para los efectos de autodiagnósticos en procesos de acreditación, en la elaboración de sus planes estratégicos y sus procesos de actualización curricular.

Así mismo, la Facultad ha empezado con la complementación de este seguimiento a través de la opinión de los empleadores de los egresados, estando a la espera de los primeros resultados que le permitirán una mayor certeza en el conocimiento del impacto de la Carrera de su Programa Académico de Contabilidad.

A través del presente artículo se busca contribuir a este conocimiento del egresado de la carrera de Contabilidad centrando la atención -esta vez- en los niveles de satisfacción profesional advertidos desde la óptica de su actual condición de egresado de una institución superior educativa pública como San Marcos, indagando específicamente en el aspecto de su situación profesional laboral actual y en la percepción sobre la pertinencia de la carrera en el medio.

En este sentido, los aspectos de satisfacción, tomados en cuenta a partir del sexo y la experiencia laboral de los egresados, fueron: satisfacción con respecto a la enseñanza recibida en la universidad, satisfacción con la remuneración que perciben en la empresa o institución donde laboran y satisfacción con respecto a las actividades que desarrollan en su centro laboral. Se tratará además de verificar las relaciones entre las variables mencionadas, considerando para una mayor exactitud en los resultados la aplicación de la prueba estadística de independencia ChiCuadrado con la cual se ha buscado determinar la relación existente entre las variables mencionadas.

Para ello se planteó la definición del problema, las variables intervinientes del mismo, el planteamiento de hipótesis y el nivel de significancia para determinar sus resultados.

Los datos obtenidos se recolectaron a través de la Encuesta del Egresado establecida por la Universidad Nacional Mayor de San Marcos aplicada por la Escuela Académico Profesional de Contabilidad en el transcurso del año 2012, período en el que se tienen registrados tres grupos de encuestados que hacen una población de 203 egresados.

\section{RESULTADOS DE LA APLICACIÓN DE ENCUESTA A LOS ALUMNOS EGRESADOS DE LA EAP DE CONTABILIDAD}

Ingreso y Egreso de la Carrera:

La población encuestada fueron los 203 egresados de la carrera de Contabilidad que 
asistieron a rendir los exámenes de titulación programados por la Facultad en el transcurso del presente año, habiéndose registrado tres grupos de egresados encuestados en las tres fechas que se indican en el cuadro siguiente.

Cuadro $N^{\circ} 01:$ Fechas de los Grupos Encuestados

\begin{tabular}{|c|c|c|}
\hline $\begin{array}{l}\text { Fecha que } \\
\text { se aplicó la } \\
\text { encuesta }\end{array}$ & Frecuencia & $\%$ \\
\hline $24-02-12$ & 75 & $36.95 \%$ \\
\hline $27-04-12$ & 67 & $33.00 \%$ \\
\hline $22-06-12$ & 61 & $30.05 \%$ \\
\hline Total & 203 & $100.00 \%$ \\
\hline
\end{tabular}

Gráfico $N^{\circ} 01$ : Año de Ingreso a la EAP de Contabilidad

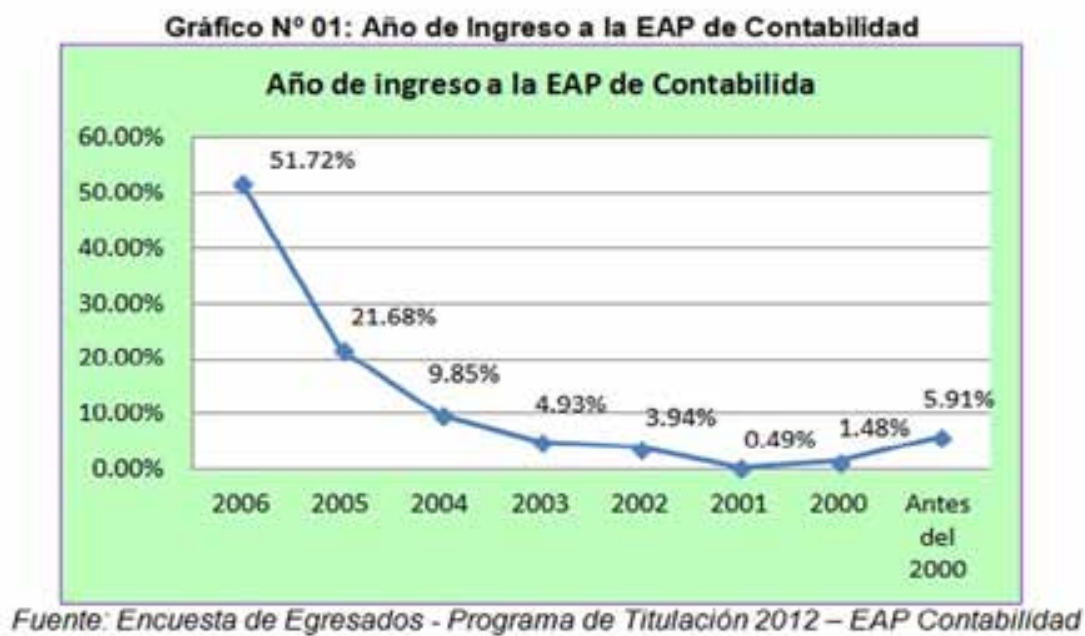

Gráfico Nº 02: Año de Egreso de la EAP de Contabilidad

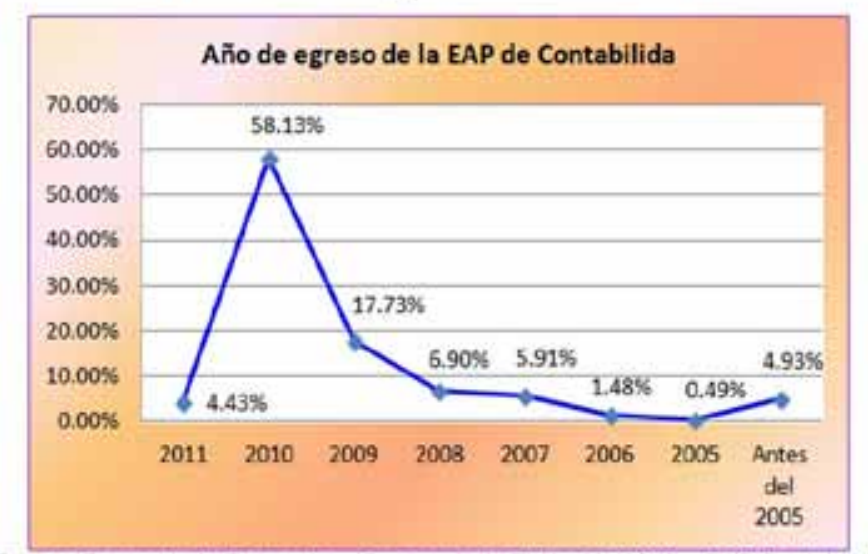

Fuente Encuesta de Egresados - Programa de Títulación 2012 - EAP Contabilidad 
De esta población encuestada se destaca que el $83.25 \%$ ingresó a estudiar la carrera entre los años 2004 y 2006. Así mismo el 87.18\% egresó de la carrera entre los años 2008 y 2011.

Otro aspecto que puede observarse es que la mayor proporción $(65 \%)$ de egresados son mujeres.

\section{Gráfico $N^{\circ} 03:$ Sexo de los Egresados encuestados}

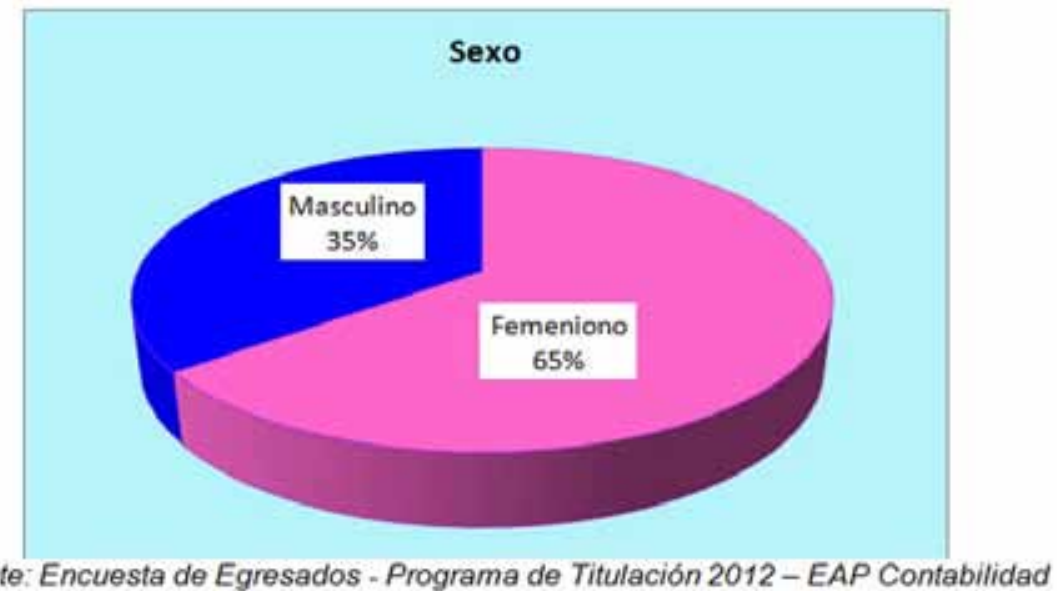

Situación Laboral de los egresados:

Al mes de junio que se aplicó la última encuesta el $91 \%$ de los egresados contaban con un empleo, lo cual constituye un nivel de empleabilidad considerablemente alto.

Gráfico $N^{\circ}$ 04: Situación Laboral de los Egresados de la EAP de Contabilidad

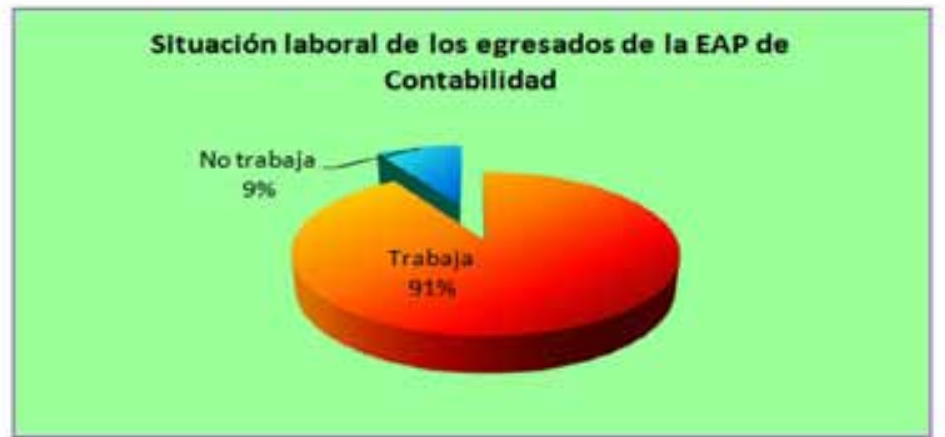

Fuente: Encuesta de Egresados - Programa de Titulación 2012- EAP Contabilidad

\section{Niveles de Satisfacción Académica y Laboral}

\section{Cuadro $N^{\circ} 02$ : Relación del Trabajo con la Carrera}

\begin{tabular}{|c|c|c|}
\hline $\begin{array}{l}\text { Relación del trabajo con la } \\
\text { carrera }\end{array}$ & Egresados & $\%$ \\
\hline $\begin{array}{l}\text { Mucho } \\
\text { Regularmente }\end{array}$ & $\begin{array}{c}159 \\
26 \\
\end{array}$ & $\begin{array}{l}85.95 \% \\
14.05 \%\end{array}$ \\
\hline Total & 185 & $100.00 \%$ \\
\hline
\end{tabular}

Fuente: Encuesta de Egresados - Programa de Titulación 2012 - EAP Contabilidad

112/ QUIPUKAMAYOC | Vol. 20(38) 2012 


\section{Cuadro $N^{\circ} 03:$ Niveles de Remuneración como Profesional}

\begin{tabular}{l|c|c}
\hline $\begin{array}{c}\text { Considera que su remuneración como } \\
\text { profesional: }\end{array}$ & Egresados & $\%$ \\
\hline ¿Mejoró su situación económica? & 165 & $89.19 \%$ \\
¿Sobrepaso su expectativa económica? & 7 & $3.78 \%$ \\
¿Sigue igual? & 13 & $7.03 \%$ \\
\hline \multicolumn{1}{c|}{ Total } & 185 & $100.00 \%$ \\
\hline
\end{tabular}

Fuente: Encuesta de Egresados - Programa de Titulación 2012 - EAP Contabilidad

\section{Cuadro $\mathrm{N}^{\circ}$ 04: Experiencia Laboral frente a Formación Profesional}

\begin{tabular}{l|c|c}
\hline $\begin{array}{c}\text { A partir de la experiencia laboral la } \\
\text { formación profesional que recibió es: }\end{array}$ & Egresados & $\%$ \\
\hline Muy apropiada & 56 & $30.27 \%$ \\
Apropiada & 100 & $54.05 \%$ \\
Regularmente apropiada & 29 & $15.68 \%$ \\
\hline \multicolumn{1}{c|}{ Total general } & 185 & $100.00 \%$ \\
\hline
\end{tabular}

Fuente: Encuesta de Egresados - Programa de Titulación 2012 - EAP Contabilidad

Cuadro N 05: Rango de Remuneración Mensual

\begin{tabular}{l|c|c}
\hline $\begin{array}{c}\text { Rango de la remuneración } \\
\text { mensual }\end{array}$ & Egresados & $\%$ \\
\hline Menos de S/ 1,000 & 19 & $10.27 \%$ \\
Entre S/ 1,001 y S/ 2,000 & 72 & $38.92 \%$ \\
Entre S/ 2,001 y S/ 3,000 & 70 & $37.84 \%$ \\
Mayor a S/ 3,000 & 24 & $12.97 \%$ \\
\hline \multicolumn{1}{c|}{ Total } & 185 & $100.00 \%$ \\
\hline Fuente: Encuesta de Egresados - Programa de Tifulación 2012-EAP Contabilidad
\end{tabular}

\section{Cuadro $\mathbf{N}^{\circ} 06$ : Orientación de la Especialidad en el Desempeño Laboral}

\begin{tabular}{l|c|c}
\hline $\begin{array}{c}\text { Si al egresar, se encontraba } \\
\text { laborando, su desempeño era: }\end{array}$ & Egresados & $\%$ \\
\hline En la especialidad & 167 & $90.27 \%$ \\
A fin/relacionado con la & 15 & $8.11 \%$ \\
especialidad & 1 & $0.54 \%$ \\
Otro diferente & 2 & $1.08 \%$ \\
\hline No estaba laborando & 185 & $100.00 \%$ \\
\hline \multicolumn{1}{c|}{ Total general } &
\end{tabular}

Fuente: Encuesta de Egresados - Programa de Titulación 2012 - EAP Contabilidad

\section{Cuadro $\mathrm{N}^{\bullet}$ 07: Niveles de Satisfacción en el Desempeño Laboral}

\begin{tabular}{l|c|c}
\hline $\begin{array}{c}\text { Respecto a las actividades que } \\
\text { viene realizando en su empleo. } \\
\text { Usted se siente: }\end{array}$ & Egresados & $\%$ \\
\hline \hline Medianamente satisfecho & 36 & $19.46 \%$ \\
Satisfecho & 123 & $66.49 \%$ \\
Muy satisfecho & 26 & $14.05 \%$ \\
\hline \multicolumn{1}{c|}{ Total general } & 185 & $100.00 \%$ \\
\hline
\end{tabular}

Fuente: Encuesta de Egresados - Programa de Titulación 2012 - EAP Contabilidad 


\section{RESULTADOS DEL ANÁLISIS \\ ESTADÍSTICO - PRUEBA \\ ESTADİSTICA CHI CUADRADO DE \\ PEARSON - A LOS EGRESADOS}

\section{ASPECTOS METODOLÓGICOS:}

\section{Prueba de Hipótesis:}

Para probar las hipótesis se utilizó la Prueba Estadística Chi Cuadrado de Pearson.

Prueba de independencia (Chi-cuadrado)

La prueba de independencia Chi-cuadrado de Pearson, nos permite determinar si existe una relación entre dos variables categóricas.

Esta prueba nos indica si existe o no una relación entre las variables, pero no indica el grado o el tipo de relación; es decir, no indica el porcentaje de influencia de una variable sobre la otra o la variable que causa la influencia.

\section{Para hallar la prueba de hipótesis:}

Plantear las hipótesis en positivo y negativo ( $\mathrm{H} 0, \mathrm{H} 1)$

Establecer el nivel de significancia: $\alpha=0.05$, que indica que el estudio esta validado a un $95 \%$ de confianza

Estadística de prueba: Chi cuadrado.

Determinar la región rechazo y región de aceptación: Se calculará el valor crítico que permita encontrar la región de aceptación y la región de rechazo:

\section{Conclusión.}

$\mathrm{Si}$ el valor de significancia es menor que alfa se rechaza $\mathrm{H} 0$ y se acepta $\mathrm{H} 1$ (si $\rho \leq 0.05)$, caso contrario se acepta $\mathrm{H} 0$.
Si rechazamos $\mathrm{HO}$ concluimos que las variables $\mathrm{X}$ e $\mathrm{Y}$ no son independientes, es decir X e Y están relacionadas.

Nota: El nivel de significancia que se establece normalmente es 0.05 , esto puede variar en la regla de decisión a 0.01 y 0.001 si se requiere mayor certeza en la prueba de hipótesis.

\section{RESULTADOS}

A PARTIR DE LA EXPERIENCIA LABORAL: EXISTE RELACIÓN ENTRE EL GRADO DE SATISFACCIÓN DE LA ENSEÑANZA RECIBIDA EN LA UNIVERSIDAD Y EL SEXO.

Variables: Sexo y grado de satisfacción de la enseñanza recibida en la universidad.

\section{Planteamiento de Hipótesis:}

Ho: A partir de la experiencia laboral: No existe relación entre el grado de satisfacción de la enseñanza recibida en la universidad y el sexo, es decir el sexo no influye en la grado de satisfacción de la enseñanza recibida en la universidad.

$\mathrm{H} 1$ : A partir de la experiencia laboral: Existe relación entre el grado de satisfacción de la enseñanza recibida en la universidad y el sexo, es decir el sexo influye en la grado de satisfacción de la enseñanza recibida en la universidad.

Nivel de significancia: $\alpha=0.05$

Se observa que la mayor proporción (40\%) de egresados son mujeres quienes opinan que la enseñanza universitaria es apropiada, pudiendo inferirse que la opinión de una enseñanza apropiada en la universidad está asociada al sexo femenino. 
Con los resultados del Chi-Cuadrado se observa que el valor de $\rho$ es menor que 0.05 , por lo tanto se acepta la hipótesis alterna: A partir de la experiencia laboral: "Existe relación entre el grado de satisfacción de la enseñanza recibida en la universidad y el sexo, es decir el sexo influye en la grado de satisfacción de la enseñanza recibida en la universidad”.

\section{Prueba estadística:}

Cuadro $\mathrm{N}^{\circ} 08$ : Grado de satisfacción de la enseñanza universitaria según Sexo

\begin{tabular}{|c|c|c|c|c|}
\hline \multirow{2}{*}{$\begin{array}{l}\text { Grado de satisfacción de la } \\
\text { enseñanza universitaria }\end{array}$} & & \multicolumn{2}{|c|}{ SEXO } & \multirow[b]{2}{*}{ Total } \\
\hline & & Mujeres & Hombres & \\
\hline \multirow[t]{2}{*}{ Muy apropiada } & Recuento & 28 & 28 & 5 \\
\hline & $\%$ del total & $15.1 \%$ & $15.1 \%$ & $30.3 \%$ \\
\hline \multirow[t]{2}{*}{ Apropiada } & Recuento & 74 & 26 & 100 \\
\hline & $\%$ del total & $40.0 \%$ & $14.1 \%$ & $54.1 \%$ \\
\hline \multirow{4}{*}{$\begin{array}{l}\text { Regularmente } \\
\text { apropiada }\end{array}$} & Recuento & 18 & 11 & 29 \\
\hline & $\%$ del total & $9.7 \%$ & $5.9 \%$ & $15.7 \%$ \\
\hline & Recuento & 120 & 65 & 185 \\
\hline & $\%$ del total & $64.9 \%$ & $35.1 \%$ & $100.0 \%$ \\
\hline
\end{tabular}

Fuente: Encuesta de Egresados - Programa de Titulación 2012 - EAP Contabilidad

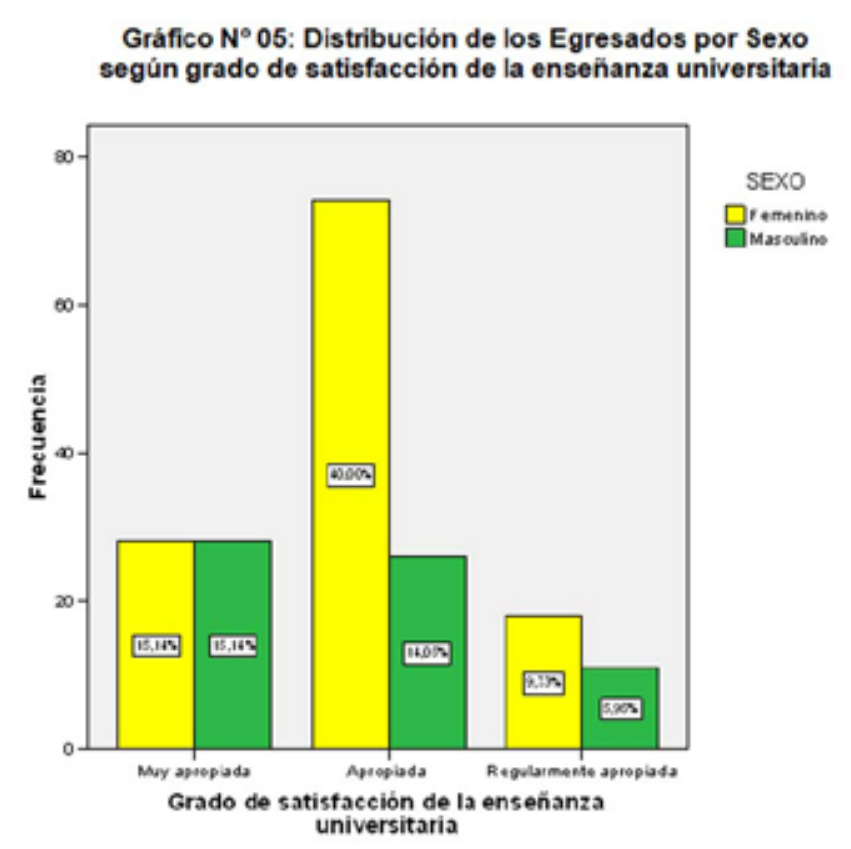

Fuente: Encuesta de Egresados - Programa de Titulación 2012 - EAP Contabilidād

Cuadro $\mathrm{N}^{\circ}$ 09: Prueba de Chi Cuadrado de Pearson

\begin{tabular}{|c|c|c|c|}
\hline & Valor & $\mathrm{gl}$ & $\begin{array}{l}\text { Sig. asintótica } \\
\text { (bilateral) }\end{array}$ \\
\hline Chi-cuadrado de Pearson & $9.191^{8}$ & 2 & .010 \\
\hline Razón de verosimilitudes & 9.123 & 2 & .010 \\
\hline $\mathrm{N}$ de casos válidos & 185 & & \\
\hline
\end{tabular}

a. 0 casillas $(.0 \%)$ tienen una frecuencia esperada inferior a 5. La frecuencia minima esperada es 10.19. 
LOS INGRESOS SALARIALES QUE PERCIBEN LOS EGRESADOS DE LA EAP DE CONTABILIDAD DIFIEREN EN LOS HOMBRES Y MUJERES.

Variables: Sexo y rango salarial que perciben los egresado de la EAP de Contabilidad.

\section{Planteamiento de Hipótesis:}

Ho: No existen diferencias salariales entre la proporción de hombres y mujeres

H1: Existen diferencias salariales entre la proporción de hombres y mujeres.

\section{Planteamiento de Hipótesis:}

Ho: "No existe relación entre los rangos salariales que perciben los egresados de la EAP de Contabilidad y el sexo, es decir el sexo no influye en los rangos salariales que perciben los egresado de la EAP de Contabilidad”.

$\mathrm{H} 1$ : "Existe relación entre los rangos salariales que perciben los egresados de la EAP de Contabilidad y el sexo, es decir el sexo influye en los rangos salariales que perciben los egresado de la EAP de Contabilidad".

Nivel de significancia: $\alpha=0.05$

\section{Prueba estadística:}

Se observa que la mayor proporción (49.2\%) de egresados son mujeres, el 29.2\% de ellas tienen un ingreso mensual entre S/. 1001.00 y S/. 2000.00 soles y el $20 \%$ tiene un ingreso de entre S/. 2001.00 y S/. 3000.00 soles.

\begin{tabular}{|c|c|c|c|c|c|}
\hline & \multirow{2}{*}{ Rango Salarial } & & \multicolumn{2}{|c|}{ SEXO } & \multirow[b]{2}{*}{ Total } \\
\hline & & & Mujeres & Hombres & \\
\hline \multirow[t]{8}{*}{$\overline{P 21}$} & Menos de S/1,000 & Recuento & 17 & 2 & 19 \\
\hline & & $\%$ del total & $92 \%$ & $1.1 \%$ & $10.3 \%$ \\
\hline & Entre $S / 1,001$ y S/ & Recuento & 54 & 18 & 72 \\
\hline & 2,000 & $\%$ del total & $292 \%$ & $9.7 \%$ & $389 \%$ \\
\hline & Entre S/2,001 y S/ & Recuento & 37 & 33 & 70 \\
\hline & 3,000 & $\%$ del total & $200 \%$ & $178 \%$ & $37.8 \%$ \\
\hline & Mayor a S/ 3,000 & Recuento & 12 & 12 & 24 \\
\hline & & $\%$ del total & $65 \%$ & $6.5 \%$ & $13.0 \%$ \\
\hline \multirow[t]{2}{*}{ Total } & & Recuento & 120 & 65 & 185 \\
\hline & & $\%$ del total & $64.9 \%$ & $35.1 \%$ & $100.0 \%$ \\
\hline
\end{tabular}

Del gráfico podríamos inferir que el sexo femenino percibe mejores ingresos que el sexo masculino.

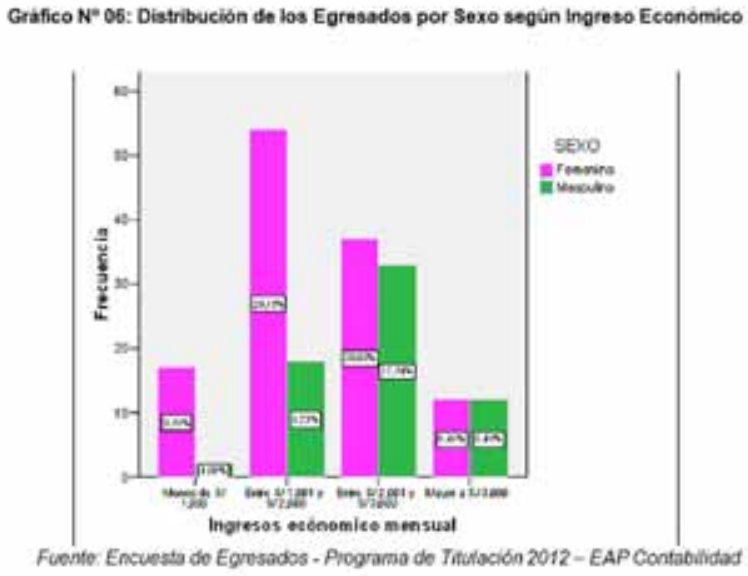

116/ QVIPURAMAYOC | Vol. 20(38) 2012 


\section{Cuadro $\mathrm{N}^{0} 11$ :}

Pruebas de chi-cuadrado

\begin{tabular}{|c|c|c|c|}
\hline & Valor & gl & $\begin{array}{l}\text { Sig. asintótica } \\
\text { (bilateral) }\end{array}$ \\
\hline Chi-cuadrado de Pearson & $15.049^{a}$ & 3 & .002 \\
\hline Razón de verosimilitudes & 16.017 & 3 & .001 \\
\hline $\mathrm{N}$ de casos válidos & 185 & & \\
\hline
\end{tabular}

a. 0 casillas $(.0 \%)$ tienen una frecuencia esperada inferior a 5 . La frecuencia mínima esperada es 6.68 .

Con los resultados del Chi-Cuadrado se observa que el valor de $\rho$ es menor que 0.05 , por lo tanto se acepta la hipótesis alterna: "Existe relación entre los rangos salariales que perciben los egresados de la EAP de Contabilidad y el sexo, es decir el sexo influye en los rangos salariales”.

\section{EXISTE RELACIÓN ENTRE EL GRADO DE SATISFACCIÓN RESPECTO A LAS ACTIVIDADES QUE DESARROLLA EN LA EMPRESA Y EL SEXO.}

Variables: Sexo y grado de satisfacción respecto a las actividades que desarrolla en la empresa. Planteamiento de Hipótesis:

Ho: No existe relación entre el grado de satisfacción respecto a las actividades que desarrolla en la empresa y el sexo, es decir el sexo no influye en la grado de satisfacción respecto a las actividades que desarrolla en la empresa.

H1: Existe relación entre el grado de satisfacción respecto a las actividades que desarrolla en la empresa y el sexo, es decir el sexo influye en la grado de satisfacción respecto a las actividades que desarrolla en la empresa.

Nivel de significancia: $\alpha=0.05$

Cuadro $\mathrm{N}^{\circ}$ 12:

Pregunta 26 Tabla Encuesta Egresados del Pregrado de la UNMSM según Sexo

\begin{tabular}{|lll|r|r|r|}
\hline & & \multicolumn{2}{|c|}{ SEXO } & \multicolumn{1}{c|}{} \\
\cline { 4 - 5 } & & $\mathrm{F}$ & $\mathrm{M}$ & \multicolumn{1}{c|}{ Total } \\
\hline P26 & Medianamente & Recuento & 22 & 14 & 36 \\
& satisfecho & \% del total & $11.9 \%$ & $7.6 \%$ & $19.5 \%$ \\
& Satisfecho & Recuento & 82 & 41 & 123 \\
& & \% del total & $44.3 \%$ & $22.2 \%$ & $66.5 \%$ \\
& Muy satisfecho & Recuento & 16 & 10 & 26 \\
& & \% del total & $8.6 \%$ & $5.4 \%$ & $14.1 \%$ \\
Total & & Recuento & 120 & 65 & 185 \\
& & \% del total & $64.9 \%$ & $35.1 \%$ & $100.0 \%$ \\
\hline
\end{tabular}

Fuente: Encuesta de Egresados - Programa de Titulación 2012 - EAP Contabilidad 


\section{Prueba estadística:}

Se observa que la mayor proporción (44.3\%) de egresados son mujeres, y se encuentran satisfechos con las actividades que vienen desarrollando en su trabajo.

Del gráfico podríamos inferir respecto a las actividades que vienen desarrollando en su trabajo el sexo femenino es satisfactorio.

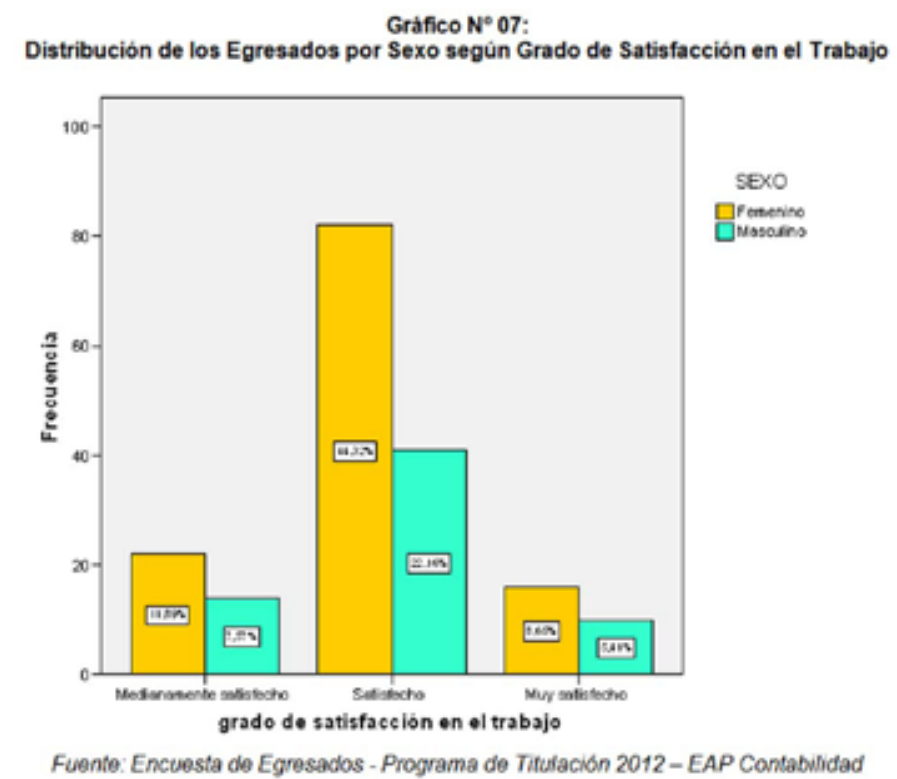

Cuadro $\mathrm{N}^{\circ} 13$

Pruebas de chi-cuadrado

\begin{tabular}{|l|c|r|r|}
\hline & Valor & gl & $\begin{array}{c}\text { Sig. asintótica } \\
\text { (bilateral) }\end{array}$ \\
\hline Chi-cuadrado de Pearson & $.524^{2}$ & 2 & .770 \\
Razón de verosimilitudes & .520 & 2 & .771 \\
N de casos válidos & 185 & & \\
\hline
\end{tabular}

a. 0 casillas $(.0 \%)$ tienen una frecuencia esperada inferior a 5 . La frecuencia mínima esperada es 9.14

Con los resultados del Chi-Cuadrado se observa que el valor de $\rho$ es mayor que 0.05 , por lo tanto se acepta la hipótesis nula: No existe relación entre el grado de satisfacción respecto a las actividades que se desarrolla en la empresa y el sexo.

\section{EXISTE RELACIÓN ENTRE EL TIEMPO QUE ESTA LABORANDO EN LA EMPRESA Y EL SEXO.}

Variables: Sexo y tiempo que esta laborando en la empresa.

\section{Planteamiento de Hipótesis:}

Ho: No existe relación entre el tiempo que esta labrando en la empresa y el sexo, es decir el sexo no influye en el tiempo que esta laborando en la empresa.

H1: Existe relación entre el tiempo que esta labrando en la empresa y el sexo, es decir el sexo influye en el tiempo que esta laborando en la empresa.

118/ QUIPUKAMAYOC | Vol. 20(38) 2012 
Nivel de significancia: $\alpha=0.05$

\section{Prueba estadística:}

Cuadro $\mathrm{N}^{0} 14$

Tiempo de Permanencia Laboral en la Empresa según Sexo

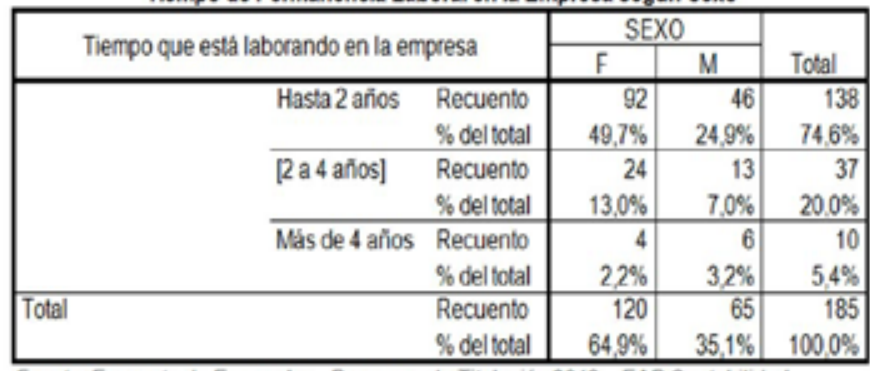

Fuente: Encuesta de Egresados - Programa de Titulación 2012-EAP Contabilidad

Se observa que la mayor proporción (49.7\%) de egresados son mujeres, y se encuentran laborando dos años en la empresa.

\section{Gráfico Nº8: Tiempo de Permanencia Laboral en la Empresa según Sexo}

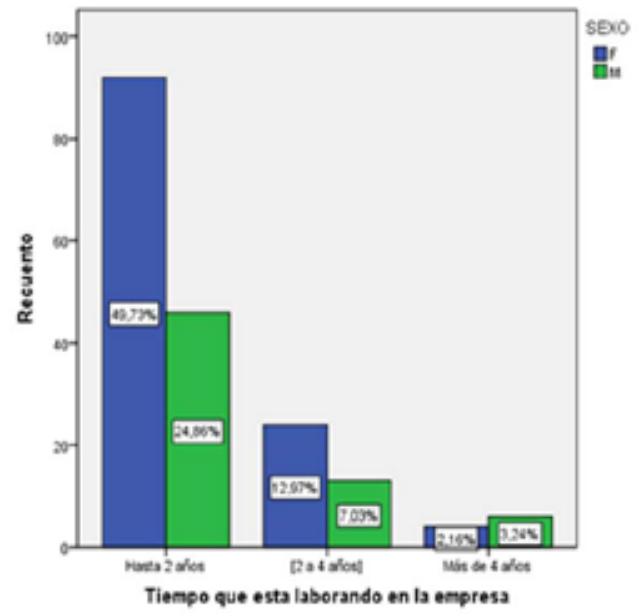

Fuente: Encuesta de Egresados - Programa de Titulación 2012 - EAP Contabilidad

Del gráfico podríamos deducir que la mayor proporción (49.73\%) de egresados son mujeres y llevan dos años trabajando en su empresa.

Prueba de Chi-Cuadrado

\begin{tabular}{|l|r|r|r|}
\hline & Valor & gl & \multicolumn{1}{|c|}{$\begin{array}{c}\text { Sig. asintótica } \\
\text { (bilateral) }\end{array}$} \\
\hline Chi-cuadrado de & $2,909^{\circ}$ & 2 &, 233 \\
Pearson & & & \\
Razón de verosimilitudes & 2,752 & 2 &, 253 \\
N de casos válidos & 185 & & \\
\hline
\end{tabular}

a. 1 casillas $(16.7 \%)$ tienen una frecuencia esperada inferior a 5 .

La frecuencia mínima esperada es 3.51 .

Con los resultados del Chi-Cuadrado se observa que el valor de $\rho$ es mayor que 0.05 , por lo tanto se acepta la hipótesis nula: "No existe relación entre el tiempo que esta laborando en la empresa y el sexo". 


\section{QUÉ RELACIÓN EXISTE ENTRE EL GRADO DE SATISFACCIÓN DE LA ENSEÑANZA RECIBIDA EN LA UNIVERSIDAD Y EL GRADO DE SATISFACCIÓN RESPECTO A LAS ACTIVIDADES QUE DESARROLLAN EN LA EMPRESA.}

Variables: Grado de satisfacción de la enseñanza recibida en la universidad y grado de satisfacción respecto a las actividades que desarrolla en la empresa.

\section{Planteamiento de Hipótesis:}

Ho: No existe relación entre el grado de satisfacción de la enseñanza que recibe en la universidad y el grado de satisfacción respecto a las actividades que desarrolla en la empresa. $\mathrm{H} 1$ : Existe relación entre el grado de satisfacción de la enseñanza que recibe en la universidad y el grado de satisfacción respecto a las actividades que desarrolla en la empresa.

Cuadro $\mathrm{N}^{\circ} 16$

Grado de Satisfacción de la Enseñanza Universitaria frente al Grado de Satisfacción respecto a las Actividades que desarrolla en la Empresa

\begin{tabular}{|c|c|c|c|c|c|}
\hline \multirow[b]{2}{*}{$\begin{array}{l}\text { Grado de satisfacción de la } \\
\text { ensefianza universitaria }\end{array}$} & & \multicolumn{3}{|c|}{$\begin{array}{c}\text { Grado de satisfacción respecto a las } \\
\text { actividades que desarrolla en la } \\
\text { empresa }\end{array}$} & \multirow[b]{2}{*}{ Total } \\
\hline & & $\begin{array}{l}\text { Medianament } \\
\text { e satisfecho }\end{array}$ & Satisfecho & $\begin{array}{c}\text { Muy } \\
\text { satisfecho }\end{array}$ & \\
\hline \multirow[t]{8}{*}{ Muy apropiada } & Recuento & 7 & 33 & 16 & 56 \\
\hline & $\%$ del total & $3.8 \%$ & $17.8 \%$ & $8.6 \%$ & $30.3 \%$ \\
\hline & Recuento & 12 & 80 & 8 & 100 \\
\hline & $\%$ del total & $6.5 \%$ & $43.2 \%$ & $4.3 \%$ & $54.1 \%$ \\
\hline & Recuento & 17 & 10 & 2 & 29 \\
\hline & $\%$ del total & $9.2 \%$ & $5.4 \%$ & $1.1 \%$ & $15.7 \%$ \\
\hline & Recuento & 36 & 123 & 26 & 185 \\
\hline & $\%$ del total & $19.5 \%$ & $66.5 \%$ & $14.1 \%$ & $100.0 \%$ \\
\hline
\end{tabular}

Fuente: Encuesta de Egresados - Programa de Titulación 2012 - EAP Contabilidad

Se observa que la mayor proporción (43.2\%) de egresados opinan que la enseñanza universitaria es apropiada y están satisfechos con las actividades que vienen desarrollando en la empresa, pudiendo deducir que una enseñanza apropiada en la universidad está asociada al nivel de satisfacción de las actividades desarrolladas en la empresa.

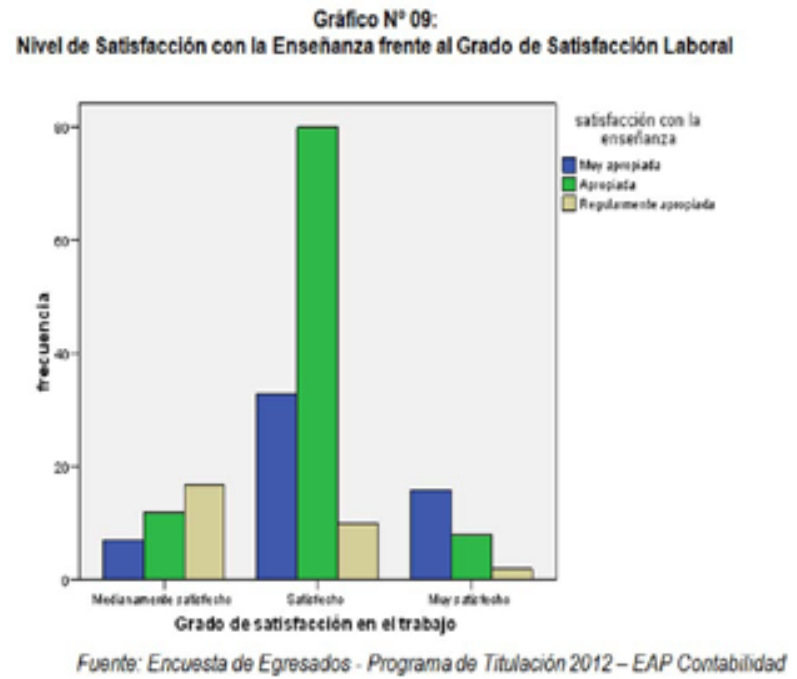

120/ QUIPURAMAYOC | Vol. 20(38) 2012 
Cuadro $\mathrm{N}^{0} 17$

Pruebas de chi-cuadrado

\begin{tabular}{|c|c|c|c|}
\hline & Valor & $\mathrm{gl}$ & $\begin{array}{l}\text { Sig. asintótica } \\
\text { (bilateral) }\end{array}$ \\
\hline Chi-cuadrado de Pearson & $46.866^{\mathrm{a}}$ & 4 & .000 \\
\hline Razón de verosimilitudes & 39.045 & 4 & .000 \\
\hline $\begin{array}{l}\text { Asociación lineal por } \\
\text { lineal }\end{array}$ & 23.940 & 1 & .000 \\
\hline $\mathrm{N}$ de casos válidos & 185 & & \\
\hline
\end{tabular}

a. 1 casillas $(11.1 \%)$ tienen una frecuencia esperada inferior a 5 . La frecuencia minima esperada es 4.08 .

Con los resultados del Chi-Cuadrado se observa que el valor de $\rho$ es menor que 0.05, por lo tanto se rechaza la hipótesis nula, es decir: "Existe relación entre el grado de satisfacción de la enseñanza que recibe en la universidad y el grado de satisfacción respecto a las actividades que desarrolla en la empresa”.

\section{CONCLUSIONES:}

1. La muestra analizada abarca un universo de egresados pertenecientes a bases de ingreso desde antes del año 2000 hasta la base 2006, los que registran egresos desde antes del 2005 hasta el 2011.

2. La mayor proporción de egresados corresponde al sexo femenino con un $65 \%$ frente a un $35 \%$ del sexo masculino.

3. Contabilidad constituye una de las carreras de mayor aceptación en el mercado pues como puede observarse, del total del universo de egresados encuestados, el $91 \%$ se encuentra en situación laboral estable.

4. Los alumnos egresados trabajan desempeñándose en su área en un $86 \%$.

5. En cuanto al nivel de remuneración el 89.19\% mejoró su situación económica, un $7 \%$ sobrepasó sus expectativas y un $13 \%$ lo considera igual.

6. En relación a la experiencia laboral frente a su formación profesional un 54.05\% opinó que fue apropiada, un $30.27 \%$ muy apropiada y un $15.68 \%$ regularmente apropiada, lo que significaría que nuestros egresados se encuentran con conocimientos suficientes para desempeñarse adecuadamente en el mercado laboral.

7. Respecto al rango de remuneración actual nuestros egresados se encuentran ranqueados entre $S / .1,000$ y 2,000 en un $38.92 \%$ y entre $S / .2000$ y $S / .3000$ en un $37.84 \%$.

8. Observando la relación entre la orientación de la especialidad en el desempeño laboral puede evidenciarse que el $90.27 \%$ de los egresados se encuentran desempeñándose en la especialidad para la cual fueron preparados y apenas un $8.11 \%$ en especialidades afines.

9. En relación a los niveles de satisfacción y desempeño laboral se concluye que un $66.49 \%$ se encuentra satisfecho, un $19.46 \%$ medianamente satisfecho y un $14.05 \%$ que refirió estar muy satisfecho, ello permite inferir que nuestros egresados constituyen un grupo humano en 
permanente desarrollo y crecimiento profesional.

De la aplicación de la Prueba Estadística de Independencia se infiere:

- Existencia de relación entre las variables: sexo y satisfacción con la formación académica recibida en la universidad.

- Existencia de relación entre las variables: sexo y satisfacción con el rango salarial percibido por los egresados.

- No hay relación entre las variables sexo: con la satisfacción de las actividades desarrolladas en la empresa.

- No hay relación entre las variables: sexo con el tiempo de permanencia en el trabajo.

- Existencia de relación entre las variables: formación profesional con las actividades desempeñadas en la empresa.

\section{REFERENCIAS BIBLIOGRÁFICAS:}

1. GUTIÉRREZ HUBY, Ana María. (2006). Artículo: Análisis Estadístico de los Egresados de la Facultad de Ciencias
Contables. Revista Quipukamayoc - Segundo Semestre. Pág.131.

2. HERNÁNDEZ ARROYO, Emil. (2006). Manual de Estadística. Recuperado de Sitio Web: http://books. google.com.pe/books?id=vahoN_YRn6QC\&pg $=\mathrm{PA} 185 \& \mathrm{lpg}=\mathrm{PA} 185 \& \mathrm{dq}=\mathrm{c}$ hi + cuadrado $\&$ source $=$ bl\&ots $=Z$ crXS YY-hs\&sig=2PVYPnEDKITDjh0Fab f33ilPbbY\&hl $=$ es \& sa $=X \&$ ei $=\mathrm{Q} 61 X U$ KHmKsP10gGV1oG4CA\&ved $=0 C D$ QQ6AEwAg\# $\mathrm{v}=$ onepage $\& \mathrm{q}=\mathrm{chi} \% 20$ cuadrado $\& \mathrm{f}=$ false

3. SARABIA ALEGRÍA, José María. PASCUAL SÁEZ, Martha. (2007). Curso Básico para Economía y Administración.

4. Recuperado de Sitio Web: http://books. google.com.pe/books?id=QEp1dROP0 $\mathrm{OAC} \&$ printsec $=$ frontcover $\& \mathrm{hl}=\mathrm{es} \# \mathrm{v}=\mathrm{o}$ nepage \&q\&f=false

5. UNIVERSIDAD NACIONAL MAYOR DE SAN MARCOS. Encuesta de Egresados de Pregrado de la UNMSM. Resolución Rectoral No 06354-R-11. Decreto Legislativo № 604. 